\title{
Deus ex machina? Demystifying rather than deifying machine learning
}

Michael Domaratzki, PhD, ${ }^{\mathrm{a}}$ and Biniam Kidane, MD, MSc ${ }^{\mathrm{b}, \mathrm{c}, \mathrm{d}}$

Supplemental material is available online.

The concept of "deus ex machina" refers to a plot device in storytelling wherein some supernatural force suddenly comes in and resolves all plot lines, often in an improbable fashion that defies the internal logic of the story up to that point. Literally meaning "god from the machine," deus ex machina draws its origins from ancient Greek theatre where actors playing gods would descend onto the stage (ostensibly from the heavens) with the aid of machines at the end of the play, thereby magically resolving all plot lines; the audience is expected to accept that all improbable resolutions were possible because the gods could do anything they wanted.

Machine learning (ML) is being increasingly used and reported in many areas, including surgery, giving the ability to make predictions for new, unseen data based on previous observations. While the use of ML is growing, there are many aspects of this new technology that are not fully understood by clinical researchers. Despite the increasing presence of large datasets suitable for ML, the use of the models can be foreign to new users. ML also has the potential to be abused if not understood; like biostatistics, care must be taken to not derive conclusions not supported by the tools. Moreover, many ML models can be opaque, in that the reasons for their predictions are not easily understood. In this review, we aim to illustrate the fundamentals of ML, the models that are commonly used, and some pitfalls; ultimately, our aim is to help clinicians and readers better understand and interpret (with caution) the growing use of ML in the literature.

One particular issue with $\mathrm{ML}$ is the increasing prevalence of computational tools that allow experimentation with

\footnotetext{
From the a Department of Computer Sciences, ${ }^{\mathrm{b}}$ Section of Thoracic Surgery, Department of Surgery, and ${ }^{\mathrm{d}}$ Department of Community Health Sciences, University of Manitoba, Winnipeg, Manitoba, Canada; and ${ }^{\mathrm{c}}$ Research Institute in Oncology and Hematology, Cancer Care Manitoba, Winnipeg, Manitoba, Canada.

Received for publication Jan 7, 2020; revisions received Feb 6, 2021; accepted for publication Feb 23, 2021; available ahead of print March 11, 2021.

Address for reprints: Biniam Kidane, MD, MSc, Health Sciences Centre, GE-611, 820 Sherbrook St, Winnipeg, Manitoba, R3A 1R9, Canada (E-mail: b.kidane@ mail.utoronto.ca).

J Thorac Cardiovasc Surg 2022;163:1131-7

$0022-5223 / \$ 36.00$

Copyright (C) 2021 by The American Association for Thoracic Surgery

https://doi.org/10.1016/j.jtcvs.2021.02.095
}

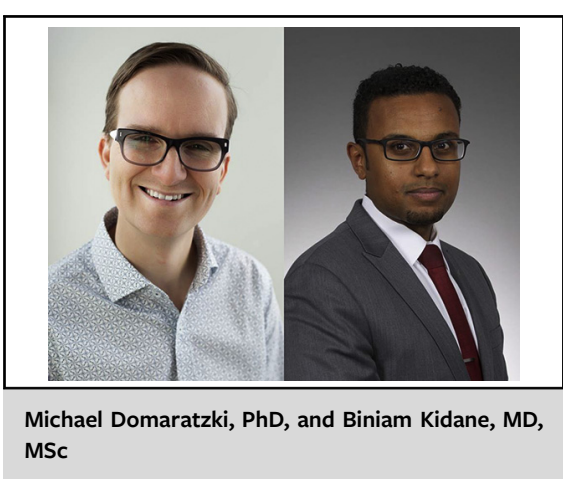

CENTRAL MESSAGE

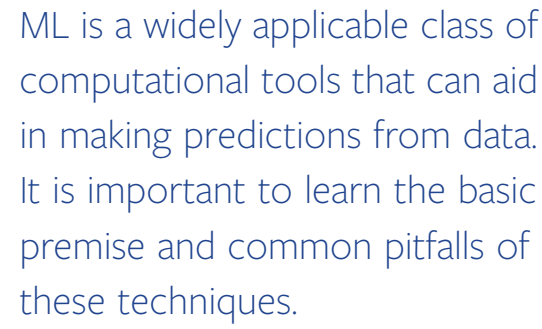

See Commentaries on pages 1138 and 1140.

models without a thorough understanding of the underlying algorithms. This potential for relatively new users to build models adds to the risk of drawing improper conclusions from data sets.

ML is a widely applicable class of computational tools that are suitable to aid in learning from existing data sets. Applications are common in many areas of society; in medicine alone, many applications have been reported in surgical outcome prediction, ${ }^{1-3}$ including cardiothoracic surgery, ${ }^{4}$ as well as in other areas such as precision medicine. $^{5-8}$ Because examples of use of ML for prediction related to cardiothoracic surgery are relatively uncommon, we have also used examples from other surgical disciplines to illustrate the concepts in this review. The same ML tools would be immediately applicable to cardiothoracic surgery as well, given suitable datasets.

ML is not a single tool, but a collection of different approaches. The selection of which approach is most suitable for a problem depends on many factors, including the availability of data, the type of data, and the type of prediction that needs to be made. In many ways, modern ML models are elaborations of regression techniques (ie, generalized linear models, generalized estimating equations) with increased computational capacity and efficiency for handling large and complex datasets, and there is no clear 
dividing line between classic regression techniques in ML. For instance, logistic regression (LR), especially stepwise techniques available on any statistical software and commonly used by researchers, is actually a form of ML. For example, some tasks described in this review such as feature selection can be used with LR as the base ML model, with positive results. ${ }^{9,10}$ This is critical in moving ML results from black box predictions to usable clinical tools. In considering whether to apply ML, it should be considered whether the problem contains sufficiently complex or large enough data to warrant ML, or whether conventional regression techniques might be more appropriate.

So when should ML be considered as a tool instead of more classic approaches such as regression analysis? No hard rule exists, and classic methods can be compared with ML methods when possible. However, when considering ML, some aspects of the problem should be kept in mind. As noted next, supervised learning techniques require a gold standard data set, and many of the techniques noted require a relatively large data set. Further, ML can be considered when the number of variables of interest is high (eg, 100s to 1000s), because ML can easily incorporate a high number of variables. Finally, as we note, the use of ML should be considered when the method for arriving at a prediction is not the primary interest. Use of ML models should also keep in mind the computational requirements for the models. In some cases, with substantial datasets and lacking specific computer hardware, training the models will be prohibitively expensive.

In this methodological review, we discuss several ML models and techniques for improving results obtained through ML. We will discuss the data in the datasets that are used to train the ML models. To avoid confusion, Table 1 defines several terms used throughout this review, which may not be familiar to readers more accustomed to traditional biostatistics.

\section{SUPERVISED VERSUS UNSUPERVISED MACHINE LEARNING}

Supervised ML is the application of a model where there is an outcome or output value that is labeled by a human supervisor. For example, providing a dataset including a wide array of different kinds of fruits with a labeled outcome variable (ie, presence of a Granny Smith apple) and example cases will allow a supervised ML algorithm/model to learn what input variables (ie, shape, color, texture, taste) predict the labeled outcome variable (ie, presence of a Granny Smith apple). It is supervised learning because the model/ algorithm is "told" what is and is not a Granny Smith apple. In unsupervised ML, there is no labeling of the outcome/ output variable, and thus the model/algorithm is not "told" what is and is not a Granny Smith apple. The unsupervised ML models/algorithms work to group together cases that share attributes with each other; they then
TABLE 1. Common terms in machine learning

\begin{tabular}{|c|c|}
\hline Term & Meaning \\
\hline Instance & $\begin{array}{l}\text { An observation or a series of observations for } 1 \\
\text { discrete individual unit of study. In datasets and } \\
\text { in the parlance of conventional statistics, this } \\
\text { would be a single row that represents the unit of } \\
\text { study (ie, a patient, a cell line, an animal, or a } \\
\text { cluster). }\end{array}$ \\
\hline Feature & $\begin{array}{l}\text { A feature is a variable of interest. It is typically } \\
\text { represented by a single column in a dataset. In } \\
\text { statistics, it is what is commonly thought of as a } \\
\text { predictor variable; however, it could very well be } \\
\text { an outcome variable as well depending on the } \\
\text { focus of the study question. In this review, we } \\
\text { refer specifically to the "outcome variable" as } \\
\text { the quantity we are interested in predicting in } \\
\text { both regression and classification tasks. }\end{array}$ \\
\hline Parameter & $\begin{array}{l}\text { A model parameter is a model variable that is } \\
\text { intrinsic to the model, and its value is derived or } \\
\text { "learned" from the available dataset. In } \\
\text { traditional regression techniques, the } \\
\text { coefficients are considered parameters. }\end{array}$ \\
\hline Hyper-parameter & $\begin{array}{l}\text { A model hyper-parameter is a "higher-level" } \\
\text { parameter that is extrinsic to the model. It is not } \\
\text { derived from the dataset. It is chosen by the } \\
\text { analyst. If one were to think of ML as a radio } \\
\text { trying to pick up a signal, hyper-parameters are } \\
\text { like the dials that can be adjusted manually by } \\
\text { the user in order to "tune" the radio. The dials (ie } \\
\text { hyper-parameters) are not part of the signal (ie, } \\
\text { data) but are a part of the machine that is trying } \\
\text { to detect and interpret the signal. }\end{array}$ \\
\hline Tuning & $\begin{array}{l}\text { Tuning is the process of varying and manipulating } \\
\text { hyper-parameters to optimize the predictive } \\
\text { performance of the intrinsic model parameters. }\end{array}$ \\
\hline
\end{tabular}

extrapolate this to new cases and try to decide which group the new case belongs to, based on which attributes these new cases possess. Thus, these unsupervised ML models/algorithms will group together green, apple-shaped objects that are crunchy and taste sour/tart together as a specific cluster. Any new case that fits these attributes is then classified as part of this cluster. Unsupervised learning can allow for discovery of relationships that were not previously identified. In traditional biostatistics, this would be akin to cluster analysis and principal component analysis.

In this review, we will only consider supervised ML techniques. Recent interest in ML has focused on supervised learning, particularly due to the introduction of deep learning, ${ }^{11}$ a relatively new class of tools that use significantly more sophisticated models (often so-called deep neural networks [DNNs]) and high computing power to learn more complex patterns in datasets. The descriptor "deep" is used because many layers of networks are used to 
transform data as you go from input to output (ie, results or prediction). In supervised learning, a training data set for the model is required. In the absence of a gold standard data set, supervised learning is not possible, and identifying an appropriate data set for supervised ML is a critical first step for researchers to determine if supervised learning can be applied. This training set consists of several instances with data on several features as well as the outcome variable. For example, in a medical setting, the training set may consist of surgical, perioperative, and demographic data (ie, the features), as well as postoperative outcome (the outcome variable), such as occurrence of a complication or death. Data for several instances (several surgeries) are needed to train the model. Supervised learning can thus be considered as similar in spirit to well-known statistical modeling tools such as regression analysis, where the outcome and predictor variables as well as their states (ie, absent or present) are known and well defined. This is differentiated from unsupervised learning that might be used in cases where the categories that the instances belong to are not known. Methods in unsupervised learning include clustering techniques such as k-means clustering and data mining techniques.

The size of the training set will also affect the suitability of ML. Even when a training set exists, a small size may restrict its use. Classic ML tools, such as Random Forests $(\mathrm{RFs})^{12}$ and Support Vector Machines $(\mathrm{SVMs})^{13}$ can be applied with smaller training sets, whereas modern tools, such as DNNs, ${ }^{11}$ typically require larger training sets, with hundreds of individuals needed for training at a minimum (Appendices E1-E5 show definitions of these ML models as well as Figures 1-3). Larger data sets representing a wide variety of instances typically allow the model to generalize and improve prediction results.

On the other hand, increasing the size of the training set will also increase training time. While training times are affected by the number of features, training time is dominated by the number of instances in the training set. However, once a model is trained, obtaining new predictions for new instances is not computationally expensive. When new data were collected, predictions for these new instances can typically be obtained instantaneously.

\section{WHAT CAN MACHINE LEARNING DO?}

Supervised ML models are based on algorithms that, although computationally intensive, have been designed by humans to obtain a well-defined outcome. While many contain an element of randomness, such as selection of elements from the training set during the training algorithm, the algorithms for training can be understood and justified by humans. As with numerical computation, computers excel at performing the training of ML models quickly. There is no mystery as to how the algorithms that the ML models used for training have been designed. In this sense,

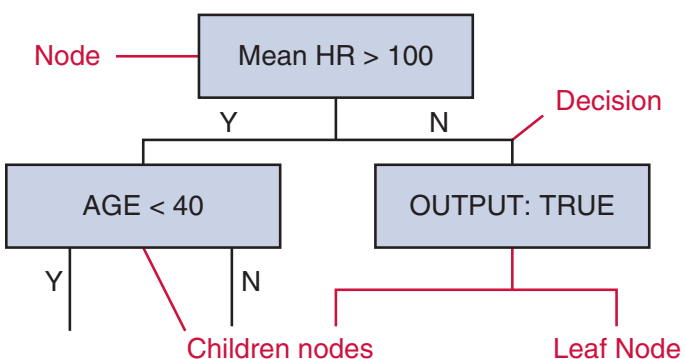

FIGURE 1. Hypothetical decision tree. Within each node is written a decision, such as "MEAN HR > 100." This is the decision for a particular feature (MEAN HR in this case) and a constant value C (100 in this case). The lines below a node (labeled $\mathrm{Y}$ and $\mathrm{N}$ ) are the paths to follow based on the outcome for that particular instance. The node on the bottom right is an example of a leaf node. When this node is reached, the decision is made for this instance. In this hypothetical example, the outcome is TRUE, which assumes we are performing binary classification, and this instance should be classified as a positive instance for the outcome under consideration. Note also that in a decision tree, the decisions in different paths are not required to have the same decisions, or even be the same length, as previous decisions will affect outcomes. Decisions in different paths are not required to have the same decisions, or even be the same length, as previous decisions will affect outcomes. $H R$, Heart rate.

ML models are transparent to human users. However, one potential drawback of ML is that although the process for learning is transparent and replicable, some of the most well-known ML algorithms do not provide any insight into the structure of the data or the features that are relevant to prediction. This is true, for instance, of DNNs. Kuhn and Johnson ${ }^{14}$ refer to this as a "tension between prediction and interpretation," which is especially present in medical fields: More accurate ML models are more complex and are thus less likely to be interpretable.

For this reason, ML models are typically viewed as black box tools ${ }^{14}$ that are better able to perform prediction than understand causality. In some cases (see "Feature Importance"), the features that are relevant to a prediction may be extracted from an ML model. However, in general, when using ML, one should expect to obtain a model where new predictions are the primary achievement, rather than insight into the structure of the complex datasets used to train the model. Recent progress in this area has developed into the field of explainable artificial intelligence, ${ }^{15,16}$ which focuses on developing tools to allow humans to understand the results obtained by ML and other artificial intelligence tools.

\section{EVALUATING MACHINE LEARNING ALGORITHMS}

To evaluate a supervised ML model, the performance of the model on a portion of the data available for training is considered. In other words, we are able to see how well the model will predict outcomes for instances where the 


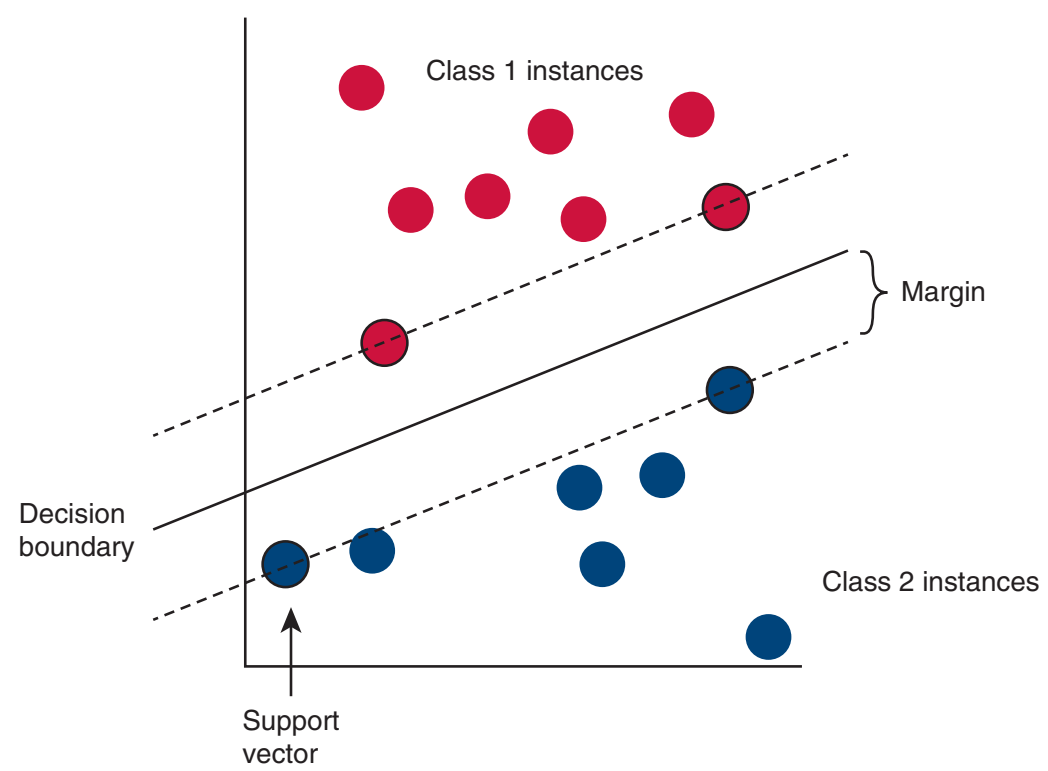

FIGURE 2. Two-dimensional representation of an SVM. In this example, each instance is represented by only 2 features, and the instances are plotted as points in a 2-dimensional plane. The 2 colors of dots represent the 2 categories to be learned by the SVM. The solid line represents the learned hyperplane (in the 2-dimensional case, a line) that classifies examples. The so-called margin is denoted by the area between the 2 dotted lines; the SVM seeks to maximize the distance between the separating hyperplane and data instances on either side of the hyperplane. The instances on the dotted lines are called the support vectors.

outcome is known (but which has not been used for training). Thus, in supervised ML, analysts will take the data set that is available and divide it into separate sets, often referred to as the training set and the test set (ie, a

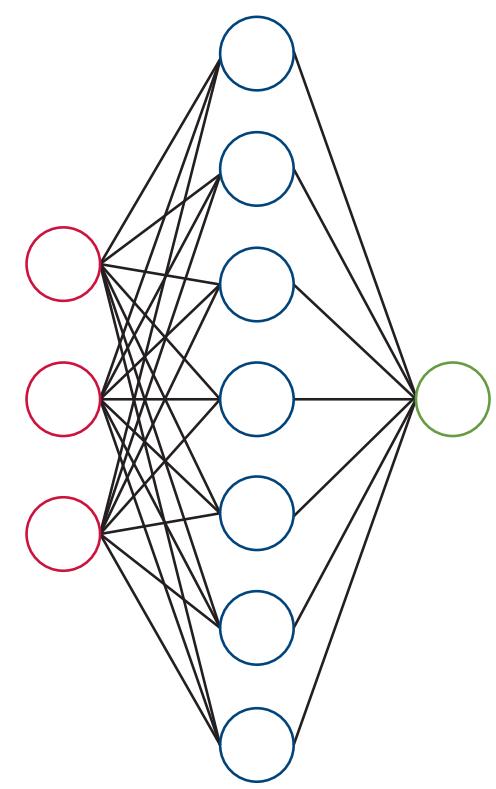

FIGURE 3. Example of a feed-forward neural network. There are 3 input nodes and 1 output node. There are also 7 nodes that are neither input nor output; these are typically referred to as hidden nodes in the context of feedforward networks. The presence of several layers of hidden nodes in a neural network is what typically characterizes that network as deep; for some tasks, a DNN can have more than 1000 hidden layers of nodes. validation set). Additionally, as part of the training, the model may require differentiation between subsets of the training set, as described in cross-validation next. ${ }^{17}$ The training set is used to train the model; this is how the model learns from existing data. The test set is not used to train the model and is instead used to evaluate the prediction of the trained model. Because the model will have not used the test set for training, it will evaluate the ability of the model to generalize its predictions to new instances and evaluate whether the model has been overfit to the training data. Overfitting is a common pitfall in conventional prediction biostatistics, and it is especially pertinent to ML because of the generally excellent capacity of many ML techniques to draw a line between predictors and outcome. A model may be perfect at predicting outcomes in a particular dataset but be useless at predicting outcomes in other datasets; this is a consequence of overfitting the model to the idiosyncrasies of the training or derivation dataset. This is why it is important to have both a training/derivation dataset and a separate test/validation dataset. This requires splitting existing datasets into 2 such datasets.

A common technique to mitigate the loss of data for creating the test set and for training ML models in general is cross-validation. In cross-validation, the entire dataset is randomly split into $k$ disjoint, equal-sized subsets, called the "folds," 18 for some integer $k>1$. Then, for each of the $k$ folds, training of the model is performed on the remaining $k$ - 1 folds, and tuning is performed on the remaining fold that 
was not used for training. This gives $k$ estimations of the accuracy of the ML model, which are averaged to give an overall view of the accuracy of the model. For classification problems, stratified cross-validation may be further used to ensure that each of the $k$ folds has a proportion of each of the levels of the outcome variable that matches the full dataset. ${ }^{18}$ When analysts use the tuning dataset as part of the test dataset (ie, validation dataset), this "double-dipping" can increase the risk of systematic errors by overfitting the model to the data used. This has the potential of compounding errors, both types I and II. Readers should keep a wary eye on the use of training, tuning, and testing sets; if they all appear to be coming from the same source (especially if it is a small dataset to begin with), this should raise a red flag.

The goal of an ML model is to minimize both type I and II errors. However, care must be taken in how to evaluate the success of an ML algorithm. An imbalanced dataset used as a training set for an ML algorithm will generally cause the model to predict the more prevalent outcome. This can inflate some typical measures of performance, such as sensitivity or specificity, depending on the imbalance. As such, measures such as F1 score, accuracy, or AUC for the ROC should be considered, especially when imbalanced datasets are present. In particular, the F1 score is considered robust in cases where a binary outcome variable appears in unbalanced proportion; it is calculated as the harmonic mean of precision (ie, TP/(TP + FP)) and recall (ie, TP/ (TP + FN), also known as "sensitivity").

In some cases, where the cost of misdiagnosis is asymmetrical, sensitivity and specificity (ie, $\mathrm{TN} /(\mathrm{TN}+\mathrm{FP})$ ) can provide insight into the relative performance of an ML model. In general, there is a classic tension between sensitivity and specificity. In some situations, it is more important to avoid incorrectly ruling out a condition than it is to be able to accurately diagnose it. This is a concept to which ML is agnostic and must be decided by the clinicians/scientists designing the ML models.

\section{MODELING INPUT}

Most ML algorithms work on a feature vector, which is a representation of instances in both the training and testing sets. A feature vector can be thought of as the combination of features in an instance, that is, a feature vector is a row of data that represent the individual of study. The feature vectors are typically the basic unit of training in ML models: Feature vectors for instances are provided to the model, which then learns from the instance. The process can be either iterative (ie, progressively refined and improved models from seeing additional inputs) or through other techniques such as repeated sampling or solving an optimization problem. In the majority of these cases, the feature vector for an instance is the basic unit of the training phase, and as the representation of an instance, the feature vectors must be consistent with each other. For example, all the feature vectors need to be the same length.

In some cases, the data that have been collected can be used directly as the feature vector, such as clinical data (eg, heart rate over time). If a fixed, consistent number of features are present for each instance, then these can be used to train the ML model. These features can be continuous numerical data or categorical data. Modern ML algorithms can handle large feature vectors with thousands of features, provided adequate computing power and training time.

However, in many situations, summarizing instances into appropriate feature vectors simplifies the analysis. One particular case where summarization may be considered is when different instances have differing amounts of data. This can occur with time series data, where the length of the data may differ between instances. In our research, for example, we explored the association between intraoperative heart rate variability and postoperative outcomes after thoracic surgery. Because of the variation in operative time, the heart rate data inputs had different lengths (ie, 1 hour worth of data vs 3 hours). Many ML algorithms, including NNs, RFs and SVMs, require all instances to have the same number of features, and in the case of uneven features, summarization of the data is necessary. For example, Prasad and colleagues, ${ }^{1}$ in predicting postoperative outcomes for orthotopic liver transplant using perioperative data such as blood pressure and heart rate, summarized time series data using the mean of the observations and the median absolute deviation.

No rules exist for designing feature vectors. Changes to the feature vector can result in significant changes to the performance of ML models. However, a general guideline is to use raw data for feature vectors. Using summarized data does not allow the ML algorithm to learn from the raw data, and as a result, it provides a chance for preconceptions about the data to be incorporated into the model. In other words, if we replace raw data in a feature vector with summary statistics, clinical scoring systems, or similar derived data, the ML model must learn from these data, which may include preconceptions or bias, rather than the raw data itself.

A major benefit of ML techniques in surgical outcomes research is the ability to model multitudinous and large amounts of intraoperative data inputs. However, these inputs themselves present modeling challenges. Cao and colleagues ${ }^{2}$ discuss the performance of ML models using data from a large Swedish bariatric surgery registry. They note that previous studies of the same dataset using multivariable LR had poor performance in prediction of postoperative complications. The authors note that preoperative predictor variables have proven to be largely insufficient for driving predictions, whether one uses ML or classic regression techniques. They note that the inclusion of intraoperative 
variables would likely improve prediction rates. However, there are 2 barriers to incorporating intraoperative variables/features to increase prediction accuracy. First, reliance on intraoperative data for model creation would preclude the ability to provide prediction at the preoperative stage; this is a major, if not prohibitive, limitation if the sole purpose of prediction tools is to inform decisions before an operation is performed. The second barrier is more of a technologic issue and relates to the difficulty of reliably capturing all important intraoperative variables and data. However, advances in intraoperative monitoring and black box technology ${ }^{16,19}$ may make it possible to capture and analyze real-time intraoperative data.

A significant aspect of preparing data for use in ML is generally known as data cleaning. This process, designed to handle inconsistencies in the data, may involve manual intervention to delete instances that contain errors or repair inconsistent data. One automated aspect of cleaning is imputation, where missing features from an instance are inferred from the dataset. Relatively elementary techniques, such as using the mode of nonmissing values for that feature, typically suffice for sufficiently detailed datasets.

\section{OVERSAMPLING AND UNDERSAMPLING}

In many medical applications, datasets will be imbalanced, without equal incidence of outcome states. For instance, in a binary outcome variable for presence/absence of a disease or a complication, we expect to have fewer positive cases than negative cases. These imbalanced datasets present challenges to ML tools; the dominant negative cases are overrepresented in the data, and ML algorithms will be inclined to predict new instances as negative ones (ie, it is easier to predict not having the disease or not having the complication). Thus, such models will have high sensitivity (with few false-negatives). However, they will have a higher risk of false-positives and thus have a moderate rather than a high degree of specificity. To solve this issue of dataset imbalance, additional tools can be applied to the dataset before the ML algorithm is trained. In Appendix E6, we describe some of these tools.

\section{TYPES OF MACHINE LEARNING ALGORITHMS}

Many different models of supervised ML algorithms exist, and it is impossible to survey all of them. In Appendices E1-E5, we describe 5 tools that take different approaches to prediction: Naive Bayes, RFs, Gradient Boosting, SVM, and Deep Learning.

These 5 models are not intended to be an exhaustive review, and many variations of the basic descriptions given here exist in the literature. In Appendices E1-E5, we describe some applications of these technologies, including predicting mortality risk in cardiac surgery patients, ${ }^{20}$ complication in cardiothoracic surgery, ${ }^{4}$ acute kidney injury after surgery, ${ }^{3}$ and esophageal cancer staging. ${ }^{21}$

\section{FEATURE IMPORTANCE}

In general, ML models are viewed as black box solutions that are incapable of yielding insight into the reasons for the predictions the model gives. However, there are some tools that can be used to gain some insight into the relative importance of the features for prediction. These tools are referred to as feature importance tools. To differentiate feature importance from feature selection, feature importance tools are generally model-specific and are used after a model is trained. The importance is derived from the strength that a feature has in aiding the model to make a prediction, so training is necessary. Feature importance tools also can be used along with feature selection tools: Statistically significant or theoretically important features can be selected before training, and the remaining features can be ranked using a feature importance tool.

Examples of techniques for feature importance are recursive feature elimination, which can be used with tools such as linear kernel SVMs, and permutation techniques, ${ }^{12}$ which can be applied more generally. In this latter technique, originally designed for RFs, a model is evaluated on data that have had some features permuted to evaluate the importance of features that are not. Variable importance (VIMP) for RFs uses impurity of nodes in trees to estimate importance for features. ${ }^{22}$ In a recent study, Wojnarski and colleagues $^{23}$ used RFs to predict aortic phenotype for 656 patients with bicuspid aortic valves. The features were 56 preoperative features, including demographic data, noncardiac morbidity, laboratory data, valve pathology, and others. The authors demonstrated through VIMP that the most relevant features are generally related to echocardiographic measurements, including peak and mean aortic valve gradient $(\mathrm{mm} \mathrm{Hg})$ and left ventricular inner diameter (diastole).

In another example, Lu and Ishwaran ${ }^{24}$ used VIMP with Cox regression to identify relevant features for predicting the risk of systolic heart failure in a dataset of 2231 patients who had undergone cardiopulmonary stress tests. The authors introduce the use of a VIMP index to replace $P$ values in determining the importance of a feature to a regression model. In predicting systolic heart failure, the VIMP index identifies peak oxygen consumption, blood urea nitrogen, and exercise time as of top importance.

\section{CONCLUSIONS}

ML techniques are being more commonly used in the medical/surgical literature. Although they are to a certain extent "black box" techniques, they can be understood and should be interpreted with caution. ${ }^{16}$ There is a growing interest in this, and some recent articles are excellent resources. ${ }^{17}$ Readers and reviewers can and should educate 
themselves on the basic premises and common pitfalls of ML techniques so that they do not fall victim to the ruse of the deus ex machina.

\section{Conflict of Interest Statement}

The authors reported no conflicts of interest.

The Journal policy requires editors and reviewers to disclose conflicts of interest and to decline handling or reviewing manuscripts for which they may have a conflict of interest. The editors and reviewers of this article have no conflicts of interest.

The authors thank Dr Jennifer M. Hensel for review of the manuscript and editing to improve flow and readability.

\section{References}

1. Prasad V, Guerrisi M, Dauri M, Coniglione F, Tisone G, De Carolis E, et al. Prediction of postoperative outcomes using intraoperative hemodynamic monitoring data. Sci Rep. 2017;7:16376.

2. Cao Y, Fang X, Ottosson J, Näslund E, Stenberg E. A comparative study of machine learning algorithms in predicting severe complications after bariatric surgery. J Clin Med. 2019;8:668.

3. Lee HC, Yoon HK, Nam K, Cho YJ, Kim TK, Kim WH, et al. Derivation and validation of machine learning approaches to predict acute kidney injury after cardiac surgery. J Clin Med. 2018;7:2018.

4. Hernandez-Suarez DF, Kim Y, Pedro Villablanca P, Gupta T, Wiley J, NievesRodriguez BG, et al. Machine learning prediction models for in-hospital mortality after transcatheter aortic valve replacement. JACC Cardiovasc Interv. 2019; 12:1328-38.

5. Bellot P, de los Campos G, Pérez-Enciso M. Can deep learning improve genomic prediction of complex human traits? Genetics. 2018;210:809-19.

6. Ho DSW, Schierding W, Wake M, Saffery R, O'Sullivan J. Machine learning SNP based prediction for precision medicine. Front Genet. 2019;10:267.

7. Montaez CAC, Fergus P, Montaez AC, Hussain A, Al-Jumeily D, Chalmers C. Deep learning classification of polygenic obesity using genome wide association study SNPs. 2018 International Joint Conference on Neural Networks (IJCNN). Available at: https://arxiv.org/abs/1804.03198. Accessed August $24,2018$.
8. Fergus P, Montanez A, Abdulaimma B, Lisboa P, Chalmers C, Pineles B. Utilising deep learning and genome wide association studies for epistatic-driven preterm birth classification in African-American women. IEEE/ACM Trans Comput Biol Bioinform. 2020;17:668-78.

9. Rajeswaran J, Blackstone E. Identifying risk factors: challenges of separating signal from noise. J Thorac Cardiovasc Surg. 2017:153:1136-8.

10. Karim M, Epi M, Tran RCL, Cochrane A, Billah B. Variable selection methods for multiple regressions influence the parsimony of risk prediction for cardiac surgery. J Thorac Cardiovasc Surg. 2017;153:1128-35.

11. LeCun Y, Bengio Y, Hinton G. Deep learning. Nature. 2015;521:436.

12. Breiman L. Random forests. Mach Learn. 2001;45:5-32.

13. Cortes C, Vapnik V. Support-vector networks. Mach Learn. 1995;20:273-97.

14. Kuhn M, Johnson K. Applied Predictive Modeling, 26. New York, NY: Springer; 2013

15. Holzinger A, Biemann C, Pattichis CS, Kell DB. What do we need to build explainable AI systems for the medical domain? Preprint. Posted online December 28, 2017. arXiv. 1712.09923.

16. Gordon L, Grantcharov T, Rudzicz F. Explainable artificial intelligence for safe intraoperative decision support. JAMA Surg. 2019;154:1064-5.

17. Liu Y, Chen CPH, Krause J, Peng L. How to read articles that use machine learning: users' guides to the medical literature. JAMA. 2019;322:1806-16.

18. Kohavi R. A study of cross-validation and bootstrap for accuracy estimation and model selection. In: IJCAI'95: Proceedings of the 14th International Joint Conference on Artificial Intelligence. Vol 2. San Francisco, CA: Morgan Kaufmann Publishers; 1995:1137-43.

19. Jung JJ, Jüni P, Lebovic G, Grantcharov T. First-year analysis of the operating room black box study. Ann Surg. 2020;271:122-7.

20. Kilic A, Goyal A, Miller J. Predictive utility of a machine learning algorithm in estimating mortality risk in cardiac surgery. Ann Thorac Surg. 2020;109: 1811-9.

21. Ishwaran H, Blackstone E, Apperson-Hanson C, Rice T. A novel approach to cancer staging: application to esophageal cancer. Biostatistics. 2009;10:603-20.

22. Breiman L, Friedman JH, Olshen RA, Stone CJ. Classification and Regression Trees. Belmont, CA: Wadsworth; 1984.

23. Wojnarski CM, Roselli EE, Idrees JJ, Zhu Y, Carnes TA, Lowry AM, et al. Machine-learning phenotypic classification of bicuspid aortopathy. J Thorac Cardiovasc Surg. 2018;155:461-9.e4.

24. Lu M, Ishwaran H. A prediction-based alternative to $\mathrm{P}$ values in regression models. J Thorac Cardiovasc Surg. 2018;155:1130-6.

Key Words: comparative effectiveness research, machine learning, statistics 


\section{APPENDIX E1. NAIVE BAYES}

Naive Bayes is a basic ML model that uses Bayes' theorem. In particular, Naive Bayes classifiers give a conditional probability of a level of the outcome variable given the features. For example, in a binary classification task, a Naive Bayes classifier will answer questions such as "given the features, what is the probability that the outcome variable is true?" The "naive" in Naive Bayes comes from the fact that because repeated applications of Bayes' theorem are needed to calculate conditional probabilities, the model assumes that the features are independent and that all features contribute equally to making a prediction. In using Bayes' theorem, the conditional probability of a particular feature given the level of the outcome variable is needed. This conditional probability can be calculated using different techniques; for features with discrete values, marginal probabilities can be used. For features with continuous values, summary statistics from the labeled training set can be calculated, and then the conditional probability can be calculated using some predefined distribution (eg, Gaussian).

Once a Naive Bayes classifier has been constructed, new instances can be classified by checking all the conditional probabilities for all levels of the outcome variable and predicting the value that has the highest probability. Naive Bayes classifiers are fast to train and perform reasonably well compared with more sophisticated ML models. For instance, Hernandez and colleagues ${ }^{4}$ studied a dataset of cardiothoracic surgery complication and found that although LR gave the top performance, Naïve Bayes performed nearly as well and similar to more complicated models such as RFs (Appendix E2).

Bayesian Networks are a more sophisticated ML model that do not need to assume independence of the features. The model is constructed by first specifying a network of nodes that are connected by directed edges (ie, implications or 1-way relationships). The nodes of the network represent a variable, typically features of the dataset. The edges of the network are the 1-way relationships between 2 nodes of the network.

\section{APPENDIX E2. RANDOM FOREST}

$\mathrm{RF}^{12}$ is an ML technique for both classification and regression that makes a prediction by combining prediction of a number of decision trees. That is, the "forest" in RF consists of individual decision trees, which are simple models with restricted predictive capacity on their own. We first describe decision trees in detail and then discuss how these decisions are combined to produce an overall prediction from the forest.

A decision tree is similar to a flowchart for decisionmaking: It is a collection of connected nodes, each of which makes a decision about a single feature. These decisions take the form of " $x_{i}>C$ " for a feature $x_{i}$ and a constant value
C. Based on the outcome of the decision at a node for a particular input, the tree proceeds to 1 of the 2 child nodes, where another decision is made. Eventually, this process leads to a leaf node, which does not have any children. Each of these leaf nodes is labeled with a class (in the case of classification) or an output value or outcome state (in the case of regression).

A portion of a hypothetical decision tree is given in Figure 1; note that the nodes are the squares in the diagram. Within each node is written a decision, such as "MEAN HR > 100." This is the decision for a particular feature (MEAN HR in this case) and a constant value C (100 in this case). The lines below a node (labeled $\mathrm{Y}$ and $\mathrm{N}$ ) are the paths to follow based on the outcome for that particular instance. The node on the bottom right is an example of a leaf node. When this node is reached, the decision is made for this instance. In this hypothetical example, the outcome is TRUE, which assumes we are performing binary classification, and this instance should be classified as a positive instance for the outcome under consideration. Note also that in a decision tree, the decisions in different paths are not required to have the same decisions, or even be the same length, because previous decisions will affect outcomes (Figure 1: hypothetical decision tree).

The power from RFs comes from using collections of decision trees. For a classification problem, the same input is used for all trees in the RF, and the decisions of all trees in the forest are collected. For classification, the majority decision of all trees is the final prediction for the input. In the case of regression, the output values for all trees are averaged to give the final output value for the forest.

To train an RF, each decision tree is trained on a different subset of the data. This prevents each decision tree from making the same prediction. For each tree, a bootstrap sample is taken of all of the instances in the training set (commonly referred to as "bagging"). A complementary technique used in RFs is feature bagging. While bagging creates different trees depending on the bootstrap sample chosen to train the tree, feature bagging selects, at each node creation step, a random subset of the features that are eligible to be selected to make the decision.

When building an RF, some values that can be adjusted (ie, hyperparameters) can affect the overall accuracy of the model for a given dataset. Most notably, these adjustable hyperparameters include the number of trees in the RF, the maximum depth of the tree, and the maximum number of features to consider at each node, and these all should be examined when training an RF model.

In particular, when training an RF, we can set an upper bound on how many different trees will be constructed in the RF. Increasing the number of trees will tend to improve the performance of the model, at the cost of increased training time. An increased number of trees does not 
guarantee a better model simply through more predictors, however. Averaging the results of the individual trees to give a final result means that additional trees may simply be duplicating the results of other trees in the RF. Rather than tuning the number of trees, Probst and Boulestieux ${ }^{\mathrm{E} 1}$ present evidence that setting the number of trees to a large but computationally feasible number is also suitable.

The maximum depth of trees of the RF can also be tuned as a hyperparameter. This represents the maximum length of the paths in the constructed trees of the forest. If the maximum depth is limited, the sets of individuals that lead to the same leaf node (where a prediction is made) may contain several different outcome variable levels. Increasing the maximum depth of trees may lead to more homogenous sets that arrive at a node, but at the cost of potentially overfitting the trees to the training set; this has the consequence of making the model good at predicting the outcome in the training dataset but poor at predicting the outcome in any future external datasets.

The maximum number of features to be considered at each node is also a hyperparameter that can be tuned in an RF. As noted earlier, RFs use feature bagging, where only a subset of the full set of features is used during training to calculate the decision (ie, "x_i $>C$ ") at each node being constructed. For classification tasks, popular ML analysis packages have a default setting wherein the number of features to be examined at each node is the log of the total number of features in the dataset. For regression, the default and suggested approach is that the number of features to be examined at each node comprise $25 \%$ of the total number of features in the dataset. As noted earlier, adding more features increases the similarity between trees in the forest, and thus takes away from the power of RFs.

RFs were used by Ishwaran and colleagues ${ }^{21}$ for predicting esophageal cancer staging. In a dataset of 4627 patients and 45 features, the authors showed that RFs are superior to individual decision trees for making predictions while maintaining the ability to understand the importance of features. The authors found that a forest of 1000 trees provided suitable staging prediction. For understanding feature importance, the authors used VIMP (see "Feature Importance") to identify those features that influence staging, most notably, depth of cancer invasion and number of cancer-positive lymph nodes. This use of ML led to significant revisions of cancer staging guidelines for esophageal cancer. $^{\mathrm{E} 2}$

\section{APPENDIX E3. GRADIENT BOOSTING}

Boosting is a general technique in $\mathrm{ML}$ that has proven successful in many areas. Like RFs, boosting is a process that creates a collection of classifiers. To make a prediction on an instance, the majority/average of the decisions from each classifier is taken. The difference in boosting is how the models are built. Unlike RFs, boosting algorithms do not build the individual classifiers in parallel. The models are instead built sequentially, and each model learns from the errors of the previous models.

To illustrate this sequential construction, consider Extreme Gradient Boosting (XGBoost), ${ }^{\mathrm{E} 3}$ a form of gradient boosting that uses decision trees, like RFs. After the initial tree is built, its error on the training data set is considered. When the second tree is built, it is built so that it is more likely to make a correct prediction on those instances in the training set where the first tree was incorrect. To do so, those instances are weighted more heavily in the training process. To build subsequent trees, the behavior of an instance on all previous trees is considered.

There are several implementations of the concept of boosting in the literature, including successful ML models such as XGBoost and Adaboost. ${ }^{\mathrm{E} 4}$ Kilic and colleagues ${ }^{20}$ used XGBoost to predict mortality risk for cardiac surgery patients. The authors considered 11,190 patients and 34 preoperative features such as demographic data, preoperative laboratory values, and echocardiographic data. The model was able to moderately outperform the Society of Thoracic Surgeons predicted risk of mortality (PROM). XGBoost was able to identify the most significant features for prediction. The 3 most significant were serum creatinine, weight, and age, and the authors note that the features have a reasonable agreement with the STS PROM. However, the agreement between the predicted risk from STS PROM and the XGBoost model was moderate $(\mathrm{r}=0.62)$.

In another example, a review of $11 \mathrm{ML}$ tools by Lee and colleagues $^{3}$ found that gradient boosting, using a variant of decision trees, performed best at predicting acute kidney injury after surgery.

\section{APPENDIX E4. SUPPORT VECTOR MACHINES}

$\mathrm{SVMs}^{13}$ are a method of prediction that uses linear algebra to interpret the data as points in space and then makes predictions based on relatively simple division of the space. In particular, SVMs view each individual as a vector in a high-dimensional vector space. For binary classification, the SVM attempts to construct a flat surface (a hyperplane) in this vector space that separates the 2 categories. If it is possible to construct this hyperplane, which we refer to as the "decision boundary," then the SVM will find the hyperplane that has maximal distance to those instances closest to the hyperplane. Learning the hyperplane from the data is a deterministic process; given the same data, the same hyperplane will be calculated each time by the SVM. Although we describe SVMs for classification, $\mathrm{SVM}$ regression is also possible.

A 2-dimensional example of an SVM for binary classification is given in Figure 2. In this example, each instance is represented by only 2 features, and the instances are plotted as points in a 2-dimensional plane. The 2 colors of dots represent the 2 categories to be learned by the SVM. 
The solid line represents the learned hyperplane (in the 2-dimensional case, a line) that classifies examples. This is the decision boundary. The so-called margin is denoted by the area between the 2 dotted lines; the SVM seeks to maximize the distance between the separating hyperplane and the data instances on either side of the hyperplane. The instances on the dotted lines are called the support vectors (Figure 2: 2-dimensional representation of an SVM).

In many cases, no hyperplane that perfectly separates the data can be found, because the data are not linearly separable; instances of both classes are intermixed when viewed as vectors in the high-dimensional vector space. There are tools to manage this case. One is to use a slack variable that accommodates misclassifications by the hyperplane, provided that the misclassified instances are reasonably close to the hyperplane. This constant, usually referred to as "C," is one of the hyperparameters of SVMs that can be optimized. In other words, a slack variable gives the SVM the ability to not perfectly classify instances; some instances of the training data may be on the incorrect side of the hyperplane. The slack variable represents a quantity of misclassification when we view the SVM as an optimization problem: Larger values of the slack variable penalize misclassified values more, causing the training algorithm to find a decision boundary that avoids misclassifications as much as possible.

Another technique for dealing with an absence of linear separability is the use of a kernel. The kernel simulates a nonlinear mapping from the high-dimensional vector space to another (typically higher-dimensional) feature space. Common kernel families include the Gaussian radial basis functions and Polynomial Kernels, as well as the Linear Kernel, which corresponds to the case of no mapping. Although no definitive rule exists for choosing kernels, some rules of thumb exist. ${ }^{\mathrm{E} 5} \mathrm{~A}$ linear kernel is more computationally efficient than RBF or other kernels, which may influence the choice of kernel. The parameters for families of kernels can be tuned through hyperparameter optimization.

\section{APPENDIX E5. DEEP LEARNING}

Deep learning is a recent significant advancement in ML. Many recent remarkable results in ML have been driven by deep learning techniques. These techniques include a variety of tools, including DNNs, which arguably have seen the most adoption in a variety of areas. DNNs represent an idealized representation of neurons in the brain, and the prediction is the result of simulating the action of the network given an input to the network.

In terms of applicability, DNNs have emerged as a primary tool in deep learning. The concept of neural networks is not new; neural networks that function in the same way as modern DNNs have been trained on datasets since the 1970s. However, a recent confluence of events, including the development of large, labeled data sets and fast graphics processing unit (GPU) algorithms, have led to DNNs that are able to be trained on huge data sets.

Many varieties of DNNs exist, including multilayer perceptrons, convolutional neural networks, long-term short memory, and others. The general form of a neural network is a collection of nodes (the model of the neuron) and edges that connect the nodes (representing the axons). The term "deep" in DNNs typically refers to the depth of the network in terms of the maximum length of paths in the model.

A trained neural network operates by sending signals (a numerical value) from nodes through edges to other nodes. In particular, an input is provided to a set of input nodes in the neural network. Then signals pass from nodes to others. A node uses the input values from a set of nodes to calculate its own signal, which is then sent on to other nodes. After computation, an output value is produced at an output node. The value calculated by this node is the prediction for the input.

An example of this setup is shown in Figure 3. There are 3 input nodes and 1 output node. There are also 7 nodes that are neither input nor output; these are typically referred to as hidden nodes in the context of feed-forward networks. As noted earlier, the presence of several layers of hidden nodes in a neural network is what typically characterizes that network as deep; for some tasks, a DNN can have more than 1000 hidden layers of nodes (Figure 3: example of a feedforward neural network).

The calculation that occurs at a node is typically simple: The edges that feed into a node have weights, and the basic value of a node is the sum of the incoming node values multiplied by the edge weights. The power of neural networks comes from the volume of nodes and the nonlinear predictions that can take place.

Training of DNNs follows a general strategy. Initially, the neural network is given random weights to the edges. Then, to train the neural network, inputs from the training set are given to the neural network and the output is recorded. This predicted output is compared with the known, desired output. The difference between the actual and predicted output is known as the error, and the back propagation algorithm is used to adjust the weights of the edges. This weight adjustment is intended to improve prediction of the algorithm on the current input. This process is repeated with randomly chosen inputs until some criterion is met (a certain number of training iterations or a certain agreement between predicted and actual outputs).

The design of a neural network for a particular problem is not a straightforward task for those who would like to apply DNN to a problem. The number of hidden layers, nodes per layer, and connections between layers are all part of the design of a network. Different configurations will affect not only performance but also training time for the model. 
The training process for DNNs is computationally expensive; many adjustments must be made in the weights, and these adjustments are made repeatedly on training data. Fortunately, the development of GPUs and the application of them to deep learning have made this computational intensive task feasible. This is in part due to the parallelization of the training task on GPUs: The simple computation required to update the weights of nodes in a network can been structured (based on the configuration of the network) to be performed in parallel on the GPU hardware.

\section{APPENDIX E6. DATASET IMBALANCE TOOLS}

To solve this issue of dataset imbalance, we can use oversampling and undersampling tools. In this context, sampling refers to selecting individuals from the training set to train the ML model with the goal of improving prediction of the ML model.

Basic sampling techniques, such as undersampling the over-represented outcome(s), are viable techniques. The main drawback from such approaches is the potential that undersampling may remove important instances that the ML model needs to learn the over-represented class. Because the methods that many ML models use to learn from particular instances are often opaque to the end-user, it is difficult to anticipate which instances are safe to remove and which are crucial to the learning process.

Modern oversampling and undersampling techniques go beyond random selection of instances. For example, synthetic minority oversampling technique (SMOTE) ${ }^{\mathrm{E} 6, \mathrm{E} 7}$ and related techniques such as $\mathrm{ADASYN}^{\mathrm{E} 8}$ generate new artificial instances of the under-represented categories to improve classification. The new artificial instances are used to improve the ML classifier and are used exclusively in the training set. On the other hand, the testing set will consist exclusively of real data instances.

As noted, these sampling techniques are useful to deal with rare instances in medical examples. For instance, Cao and colleagues ${ }^{2}$ examined a set of more than 44,000 bariatric surgery patients and studied 29 different ML algorithms to predict severe complications from 16 preoperative features, including traits linked to obesity. However, there was a substantial data imbalance because only $3.2 \%$ of patients had severe complications after surgery. Of the 29 different ML techniques examined, the authors found that those performing with suitable sensitivity and specificity were generally methods trained using SMOTE-augmented training sets. One advantage of sampling techniques is that they are independent of the ML algorithm used; in other words, sampling techniques like SMOTE are independent of the ML technique used to make predictions.

However, synthetic oversampling techniques in particular are still heuristic algorithms. Such heuristics are not guaranteed to increase performance, and caution should be exercised when using these techniques. In particular, performance of these approaches should, at the least, be compared with performance on datasets that have not been augmented with synthetic instances.

\section{E-References}

E1. Probst P, Boulestieux AL. To tune or not to tune the number of trees in Random Forest. J Mach Learn Res. 2018;18:1-18.

E2. Rice T, Blackstone E, Rusch V. A cancer staging primer: esophagus and esophagogastric junction. J Thorac Cardiovasc Surg. 2010;139:527-9.

E3. Chen T, Guestrin C. XGBoost: A Scalable Tree Boosting System. In: KDD'16. Proceedings of the 22nd ACM SIGKDD International Conference on Knowledge Discovery and Data Mining. New York, NY: Association for Computing Machinery; 2016:785-94.

E4. Freund Y, Schapire R. A decision-theoretic generalization of on-line learning and an application to boosting. J Comput Syst Sci. 1997;55:119-39.

E5. Hsu CW, Chang CC, Lin CJ. A practical guide to support vector classification [technical report]. Taipei, Taiwan: Department of Computer Science, National Taiwan University; 2003. Available at: https://www.researchgate.net/profile/ Chenghai-Yang/publication/272039161_Evaluating_unsupervised_and_supervi sed_image_classification_methods_for_mapping_cotton_root_rot/links/55f2c574 08ae0960a3897985/Evaluating-unsupervised-and-supervised-image-classification -methods-for-mapping-cotton-root-rot.pdf. Accessed April 1, 2021.

E6. Fernández A, Garcia S, Herrera F, Chawla NV. SMOTE for learning from imbalanced data: progress and challenges, marking the 15-year anniversary. J Artif Intell Res. 2018;61:863-905.

E7. Chawla NV, Bowyer KW, Hall LO, Kegelmeyer WP. SMOTE: synthetic minority over-sampling technique. J Artif Intell Res. 2002;16:321-57.

E8. He H, Bai Y, Garcia EA, Li S. ADASYN: Adaptive synthetic sampling approach for imbalanced learning. In: IEEE International Joint Conference on Neural Networks, 2008: IJCNN 2008 (IEEE World Congress on Computational Intelligence). Piscataway, NJ: IEEE; 2008:1322-28. 